\title{
Histopathological Validation of the Surface-Intermediate-Base Margin Score for Standardized Reporting of Resection Technique during Nephron Sparing Surgery
}

\author{
Andrea Minervini, ${ }^{*}$ Riccardo Campi, Alexander Kutikov, Ilaria Montagnani, \\ Francesco Sessa, Sergio Serni, Maria Rosaria Raspollini and Marco Carini \\ From the Department of Urology (AM, RC, FS, SS, MC) and Department of Pathology (IM, MRR), University of Florence, Careggi \\ Hospital, Florence, Italy, and Division of Urologic Oncology, Fox Chase Cancer Center, Philadelphia, Pennsylvania (AK)
}

\begin{abstract}
Abbreviations
and Acronyms

$\mathrm{HRM}=$ healthy renal margin

NSS $=$ nephron sparing surgery

$\mathrm{ORS}=$ operative resection

strategy

$\mathrm{PC}=$ pseudocapsule

$\mathrm{RT}=$ resection technique

$\mathrm{SIB}=$ surface-intermediate-base

SSA $=$ score specific area

Accepted for publication May 21, 2015

Study received institutional review board approval.

* Correspondence: Clinica Urologica I, Azienda Ospedaliera Careggi, Università di Firenze, Viale San Luca, 50134, Firenze, Italy (telephone: +390552758011， +390557949209, +3934758 65716; FAX: +390552758014 ; e-mail: andreamine@libero.it).
\end{abstract}

See Editorial on page 867.

For another article on a related topic see page 1112.

Purpose: The surface-intermediate-base margin score is a novel standardized reporting system of resection techniques during nephron sparing surgery. We validated the surgeon assessed surface-intermediate-base score with microscopic histopathological assessment of partial nephrectomy specimens.

Materials and Methods: Between June and August 2014 data were prospectively collected from 40 consecutive patients undergoing nephron sparing surgery. The surface-intermediate-base score was assigned to all cases. The score specific areas were color coded with tissue margin ink and sectioned for histological evaluation of healthy renal margin thickness. Maximum, minimum and mean thickness of healthy renal margin for each score specific area grade (surface $[\mathrm{S}]=0, \mathrm{~S}=1$; intermediate $[\mathrm{I}]$ or base $[\mathrm{B}]=0$, I or $\mathrm{B}=1$, I or $\mathrm{B}=2$ ) was reported. The Mann-Whitney $U$ and Kruskal-Wallis tests were used to compare the thickness of healthy renal margin in $\mathrm{S}=0$ vs 1 and $\mathrm{I}$ or $\mathrm{B}=0$ vs 1 vs 2 grades, respectively.

Results: Maximum, minimum and mean thickness of healthy renal margin was significantly different among score specific area grades $\mathrm{S}=0$ vs 1 , and $\mathrm{I}$ or $\mathrm{B}=0$ vs 1,0 vs 2 and 1 vs $2(\mathrm{p}<0.001)$. The main limitations of the study are the low number of the $\mathrm{I}$ or $\mathrm{B}=1$ and $\mathrm{I}$ or $\mathrm{B}=2$ samples and the assumption that each microscopic slide reflects the entire score specific area for histological analysis.

Conclusions: The surface-intermediate-base scoring method can be readily harnessed in real-world clinical practice and accurately mirrors histopathological analysis for quantification and reporting of healthy renal margin thickness removed during tumor excision.

Key Words: carcinoma, renal cell; pathology; validation studies

PARTIAL nephrectomy resection techniques vary. Surgical approaches differ not only between institutions and surgeons, but also depend on each particular tumor location and anatomical complexity. ${ }^{1}$ Indeed, the amount of normal renal parenchyma that is excised with each tumor affects complication rates, preserved parenchymal volume, surgical margin status and potentially oncological outcomes. ${ }^{2-7}$ Yet the urological literature historically has largely avoided the detailed reporting of $\mathrm{RT}$ used during NSS. ${ }^{8}$

Recently the SIB margin score was proposed as a novel reporting system to classify and communicate tumor 
RT during NSS. ${ }^{1}$ The SIB model is based on a visual analysis of the intrarenal side of the specimen (fig. 1), and proposes a structured classification system to quantify and report RT in a standardized fashion across published series (fig. 2). ${ }^{1,9}$ In the current study we evaluated the feasibility of the SIB score assessment in a real-world clinical setting and provided definitive histopathological corroboration of the scoring system's validity (histopathological validation).

\section{MATERIALS AND METHODS}

After institutional review board approval was obtained, between June and August 2014 data were prospectively collected from a cohort of 40 consecutive patients undergoing NSS for localized renal tumors at our institution. NSS was performed by 4 experienced surgeons. The ORS for tumor excision was to search and carefully develop by blunt dissection the natural cleavage plane between the $\mathrm{PC}$ and normal parenchyma.

\section{SIB Score Assignment}

A detailed, step-by step overview of SIB score assignment is shown in figure 1 . After surgery the specimen was oriented in the operating theater and visually analyzed as mutually agreed by 2 surgeons (AM, RC) according to the SIB scoring system. ${ }^{1,9}$ Discrepancies were resolved by open discussion.

For SIB score assignment the intrarenal portion of the specimen was divided into 3 approximately equivalent circumferential macro areas (step 1-delineation of the surface, intermediate and base macro areas). Then, the 3 critical areas for SIB score assignment (SSAs), defined as the macroscopically evident zones of minimal margin within each macro area (SSA-Surface, SSA-Intermediate and SSA-Base) were visually defined on the specimen (step 2-delineation of the SSAs, fig. 1). Then a visual analysis of each SSA was made by the surgeon to
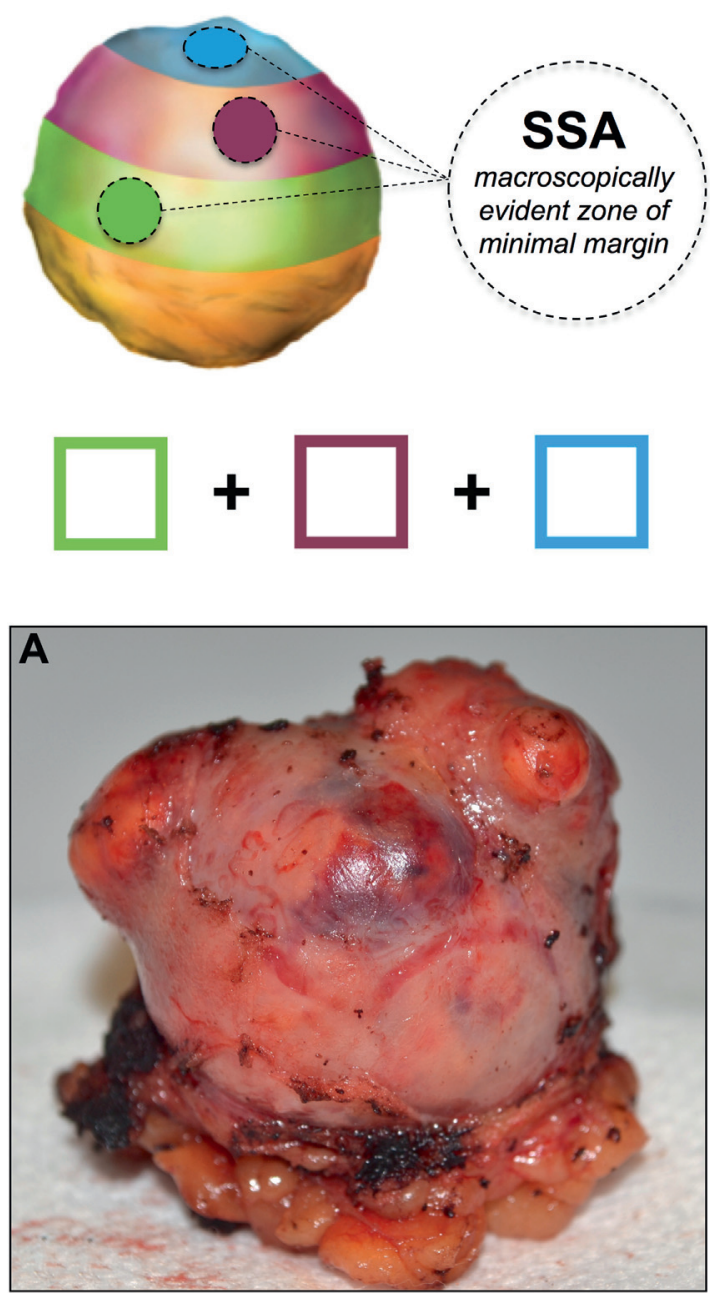

\begin{tabular}{|c|c|c|c|c|}
\hline \multirow{2}{*}{$\begin{array}{l}\text { Resection } \\
\text { technique } \\
\text { in the SSA }\end{array}$} & \multirow{2}{*}{ Visual definition } & \multicolumn{3}{|c|}{ SIB SCORE } \\
\hline & & Surface & Intermediate & Base \\
\hline Enucleation & $\begin{array}{c}\text { Only the } \\
\text { pseudocapsule is seen, } \\
\text { no additional overlying } \\
\text { tissue }\end{array}$ & 0 & 0 & 0 \\
\hline Enucleoresection & $\begin{array}{c}\text { Minimal margin of } \\
\text { parenchyma with tumor } \\
\text { contour readily visible }\end{array}$ & 1 & 1 & 1 \\
\hline Resection & $\begin{array}{c}\text { Tumor contour cannot } \\
\text { be appreciated through } \\
\text { resected parenchyma }\end{array}$ & 1 & 2 & 2 \\
\hline
\end{tabular}

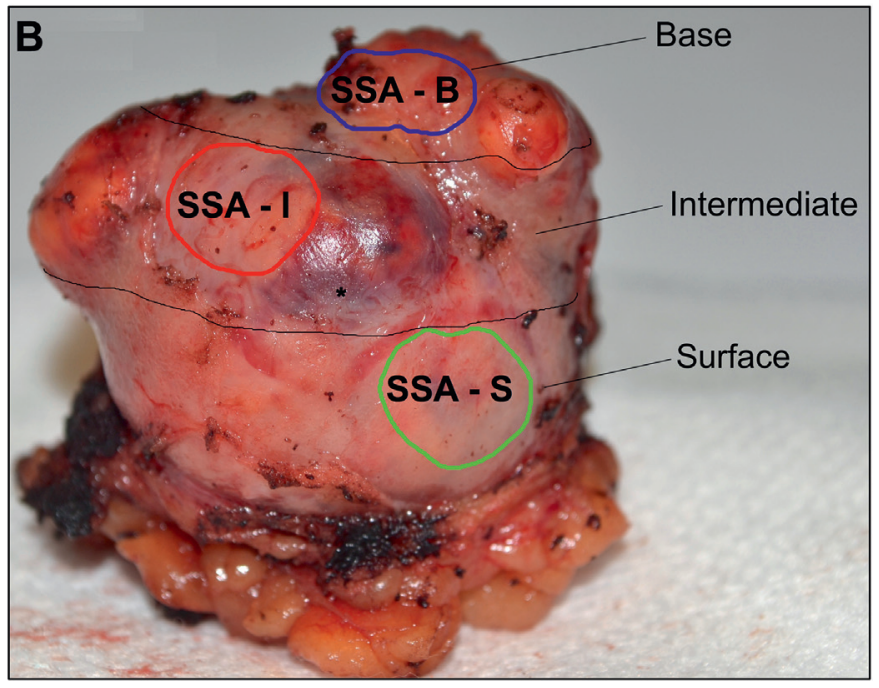

Figure 1. Identification and grading of SSA for SIB score assignment. $A$, intrarenal portion of specimen is divided into surface, intermediate and base areas. $B$, SSAs are visually assigned and defined as enucleation, enucleoresection or resection according to careful assessment of tumor contours and PC visibility. ${ }^{9}$ In case thickness of healthy renal margin is homogeneous in given macro area (S, I or B), then every zone of minimal margin in that macro area can be identified as SSA. 


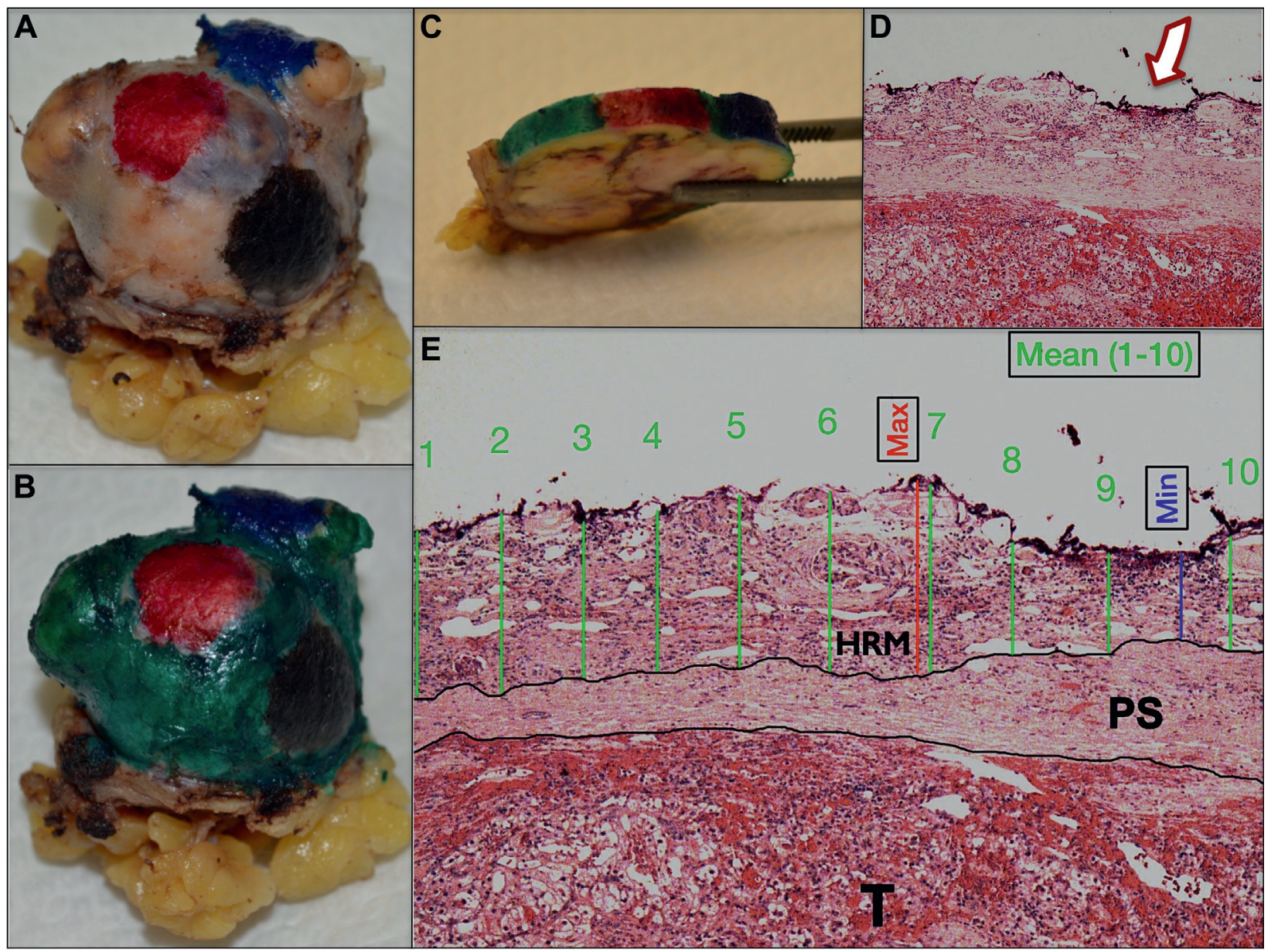

Figure 2. Overview of main steps for histopathological analysis of HRM thickness in SSAs used for SIB score validation. $A$, SSAs are inked with black, red and blue. $B$, remaining areas of intrarenal portion of specimen are inked green. $C$, central portion of each SSA is included in 1 sample for histological analysis. $D$, color of ink at outer boundary of each SSA related histological slide (white arrow) allows uropathologist to recognize SSA. $E$, histological analysis of SSA related slides at $5 \times$ magnification. Once tumor (T), peritumoral pseudocapsule (PS) and HRM are identified, maximum and minimum HRM thickness (red and blue lines, respectively) is measured. Mean HRM thickness is calculated as mean value of 10 equally spaced intervals (green lines), as shown.

determine which of the 3 definitions of resection techniques proposed by the SIB model (enucleation, enucleoresection, resection $)^{9}$ was the most appropriate to define the resection technique performed in that SSA (step 3-standardized definition of resection technique in the SSAs). Then the score was outlined by assigning zero, 1 or 2 points to each SSA depending on the resection technique appointed to that area (step 4-grading of SSAs, fig. 1). Finally, the overall RT was classified as pure enucleation, hybrid enucleation, pure enucleoresection, hybrid enucleoresection or resection according to the SIB classification system. ${ }^{1}$ Any violation of the PC (capsulotomy) was reported with the subscript "c".

\section{Digital Inking of Specimen}

In all cases a digital picture of the specimen was made after the SIB score assignment. The SSAs were digitally color-coded on the image to provide anatomical landmarks for histological analysis, with green, red and blue for the SSA-S, SSA-I and SSA-B areas, respectively (fig. 1, B).
The specimen and the digital image were then sent for pathological analysis.

\section{Handling of Specimen}

After fixation in a $10 \%$ formalin solution the specimen was handled and evaluated for appropriate sampling by a dedicated uropathologist (IM) in a completely blinded fashion. The greatest diameters of the tumors were measured and recorded. The specimen was oriented, its intrarenal portion was visually analyzed and the SSAs were identified, harnessing the digital image for guidance. SSA-S, I and B were black, red and blue inked, respectively (fig. 2, A). Any capsulotomy was reported and the remaining areas of the intrarenal side of the specimen were inked green (fig. 2, B) to analyze the surgical margin status for the whole specimen. In all cases a digital image of the inked specimen confirmed that the SSAs identified by the surgeon were included in the pathological sampling. Finally, the specimen was sectioned for histological analysis so that the central 
portion of each SSA was included on the pathological slide (fig. 2, $C$ ).

\section{Histopathological Analysis}

The histopathological analysis of the SSA related slides was done by an independent, dedicated uropathologist (MRR) in a completely blinded fashion. All cases were staged according to the 2010 TNM criteria ${ }^{10}$ and nucleolar grading was assigned according to the ISUP (International Society of Urological Pathology) grading recommendations. ${ }^{11}$ Histopathology was reviewed according to the ISUP Vancouver classification. ${ }^{12}$

The 3 SSAs were identified by the color of the ink at the upper boundaries of each slide (fig. 2, D). Maximum, minimum and mean HRM thickness beyond peritumoral PC were evaluated at $5 \times$ magnification and measured with a millimeter lens (fig. 2, E). Mean HRM thickness was calculated as the mean value of 10 equally spaced independent measures. Other variables assessed were tumor stage, histological subtype, nucleolar grade and surgical margin status.

\section{Statistical Analysis}

Statistical analyses and reporting of results were conducted according to recently published guidelines. ${ }^{13}$ Maximum, minimum and mean HRM thicknesses for each $\mathrm{SSA}$ grade $(\mathrm{S}=0, \mathrm{~S}=1$; I or $\mathrm{B}=0$, I or $\mathrm{B}=1$, I or $\mathrm{B}=2$ ) were considered the outcome variables. The SSA grades $\mathrm{I}=0$ and $\mathrm{B}=0$ were analyzed as a single category because for SIB score assignment zero, 1 or 2 points are assigned in SSA-I and SSA-B to the visual definitions of enucleation, enucleoresection and resection, respectively. The same concept was applied to SSA grade $\mathrm{I}=1, \mathrm{~B}=1$ and $\mathrm{I}=2, \mathrm{~B}=2$. Continuous variables are presented as medians and IQR while categorical variables are presented with frequencies and proportions.

Univariate analysis was used to assess the correlation between visual definitions of RTs and HRM thickness in each SSA grade at histopathological analysis. In particular, the Mann-Whitney U and Kruskal-Wallis tests were used to compare maximum, minimum and mean HRM thickness in $\mathrm{S}=0 \mathrm{vs} \mathrm{S}=1$ and $\mathrm{I}$ or $\mathrm{B}=0 \mathrm{vs} \mathrm{I}$ or $\mathrm{B}=1 \mathrm{vs}$ I or $\mathrm{B}=2$, respectively. All tests were 2 -sided with a significance level set at $\mathrm{p}<0.05$. All statistical tests were performed using SPSS ${ }^{\circledR}$ version 20.

\section{RESULTS}

Preoperative clinical data and main pathological outcomes are reported in supplementary table 1 (http://jurology.com/). For SIB score assignment, in $26(65 \%)$ patients the SSA within the surface zone was visually defined as enucleation $(S=0)$, while in $15(35 \%)$ as enucleoresection or resection $(\mathrm{S}=1)$. The SSAs in the intermediate and base zones were defined as enucleation in $29(72 \%, \mathrm{I}=0)$ and 30 $(75 \%, \mathrm{~B}=0)$, enucleoresection in $10(25 \%, \mathrm{I}=1)$ and $5(12.5 \%, \mathrm{~B}=1)$, and resection in $1(3 \%, \mathrm{I}=2)$ and 5 $(12.5 \%, \mathrm{~B}=2)$, respectively. In 1 case a capsulotomy was reported.
The overall classification of RTs in our series according to the SIB model is shown in supplementary table 2 (http://jurology.com/). HRM thickness for each SSA grade at histopathological analysis is reported in figure 3. For the surface area, HRM thickness in SSAs defined as enucleation $(S=0)$ was significantly lower than in SSAs defined as enucleoresection or resection $(S=1)$ for all histological values (maximum, minimum and mean) $(\mathrm{p}<0.001)$. Similarly, for the intermediate and base areas, HRM thickness was significantly different between SSAs defined as enucleation (I or $\mathrm{B}=0$ ), enucleoresection ( $\mathrm{I}$ or $\mathrm{B}=1$ ) and resection ( $\mathrm{I}$ or $B=2)$ for all histological values $(p<0.001)$.

\section{DISCUSSION}

Our study has proven the clinical applicability of the SIB scoring system for the standardized reporting of resection technique during NSS. Moreover we demonstrated that SIB uniform definitions of RTs, based on a visual assessment of HRM thickness, are strongly associated with histopathological assessment.

For SIB scoring, enucleation, enucleoresection, resection and capsulotomy are defined according to anatomical tumor landmarks that can be easily appreciated from the specimen. ${ }^{9}$ Our study has clearly shown that adherence to the SIB visual definitions allows for the surgeon to accurately evaluate RTs in all SSAs, mirroring a detailed histopathological grading of surgical margins. As such, the SIB definitions of enucleation, enucleoresection and resection do have a quantifiably different histopathological appearance (fig. 3). ${ }^{9,14}$ Therefore, the SIB model provides accurate and reproducible definitions of RTs that significantly mirror the gold standard of histopathological analysis. Based on such visual definitions, the novel SIB reporting system (supplementary table 2, http://jurology.com/) allows the avoidance of resource consuming assessments by the pathologist who is unfamiliar with specimen orientation, and instead affords the surgeon performing the resection a tool for rapid intraoperative quantification and communication of HRM. As such, the SIB visual definitions of RTs and the SIB scoring system significantly lower barriers for systematic HRM assessment and standardized RT reporting.

RT may influence a number of perioperative and postoperative outcomes. ${ }^{2-7}$ Thus, meaningful comparisons of published NSS series up to now have not been possible due to variability in resection techniques among institution, surgeons, tumor types and locations. Indeed, at our institution where we attempt pure enucleation for all tumors, quantification of RT using the SIB score demonstrated that heterogeneity in resection technique exists with 


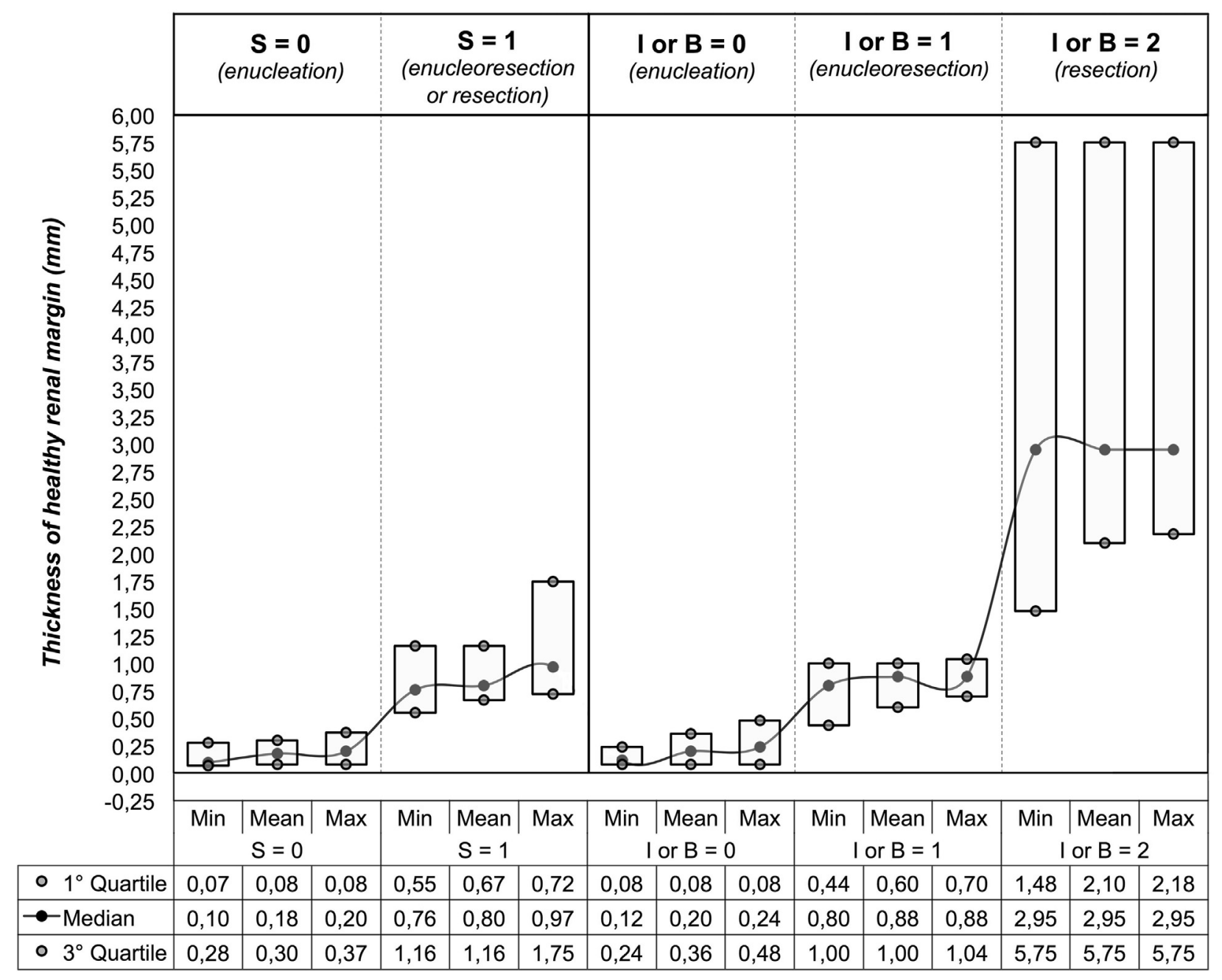

Figure 3. Box-plots showing minimum (Min), mean and maximum (Max) thickness of HRM for SSA grades $\mathrm{S}=0, \mathrm{~S}=1, \mathrm{I}$ or $\mathrm{B}=0, \mathrm{I}$ or $\mathrm{B}=1$ and $\mathrm{I}$ or $\mathrm{B}=2$. Median and IQR (white boxes) are shown.

$30 \%$ of specimens having RT definitions that do not meet the strict definition of pure tumor enucleation (supplementary table 2, http://jurology.com/). As such, including the SIB score as a variable in future reports of NSS series may allow more objective and meaningful comparisons of results between surgeons and institutions.

Since HRM thickness may not be homogeneous in the intrarenal portion of the specimen, the ability to describe and report all possible combinations of RTs, including hybrid approaches, is an important aspect of the SIB model, and allows the nuanced communication of RTs which previously was not possible. Such hybrid RTs occurred in $20 \%$ of our surgical series. Thus, the preoperative intent of the surgeon, the ORS, does not invariably reflect the actual surgical result. ORS could be conceptually classified as enucleative or resective. While in the former case the surgeon aims to develop a cleavage plane along the peritumoral PC so that no HRM is removed (pure enucleative ORS) or only a minimal layer of normally appearing parenchyma (minimal margin ORS) is removed, in the latter the surgeon follows a deeper resection plane, removing, by definition, a macroscopic layer of HRM. Although an intuitive model to communicate RTs during NSS, resection strategy cannot be used for reliable comparison and interpretation of published series as it is based on surgeon intended strategy rather than the surgical specimen.

Indeed, in our series, although a pure enucleative ORS was always attempted by the surgeon, in nearly a third of cases the RT was not classified as pure enucleation once the surgical specimen was critically examined (supplementary table 2, http:// jurology.com/). As such, a standardized reporting system that assesses the actual postoperative surgical result represents an important step toward objectification. Although a relationship between ORS and RT may exist, the SIB score overcomes an ORS based reporting approach as it considers all 
possible intraoperative deviations of planned surgical strategy and enables transparent comparisons of surgical results. Moreover, regardless of 1) surgical approach for NSS (open or minimally invasive), 2) anatomo-radiological characteristics of renal tumors (including presence of a well-defined $\mathrm{PC}$ and degree of endophytic growth) and 3) the nature of the renal mass at preoperative imaging, the SIB scoring method can be easily applied to all types of resection as the model is entirely focused on the final surgical specimen. Accordingly, resection techniques performed with open, laparoscopic or robotic approaches for exophytic, partially endophytic and even completely endophytic renal masses can be accurately classified and reported according to the SIB score.

Also, with few exceptions, such as radiologically confirmed benign angiomyolipomas (for which it might be more difficult to perform a visual assessment of the specimen according to the SIB algorithm), all renal masses eligible for NSS (including malignant tumors and renal masses of undetermined nature at preoperative imaging) should be evaluated according to the SIB score for standardized reporting of resection technique. In addition, in cases of renal tumors defined as benign on postoperative histopathological analysis, it would still be critical to report the resection technique performed during surgery since it might affect not only oncologic but also surgical and functional outcomes. Thus, our study demonstrates that the SIB model is a versatile and accurate classification system that can be easily harnessed in a real-world clinical setting.

However, the present study does have limitations. The SIB model inherently contains a certain degree of subjectivity. Thus, meticulous adherence to the SIB model steps for visual assessment of the specimen and for reporting the resection technique represents a key element to reduce the effect of this potential limitation.

In addition, the interobserver variability of SIB score assignment was not evaluated in this study. All cases reported were performed with a single technique (robot-assisted partial nephrectomy) and with an enucleative ORS. Although surgical approach and surgeon ORS should not represent critical variables for SIB score assignment, standardized reporting of resection techniques performed by different surgeons with different techniques and operative resection strategies will increase the quality of future research.

The sample size was not calculated a priori. However, although our series contained few pure enucleoresections and few hybrid enucleoresections and resections, the small sample size did not prevent assuring a highly significant association between HRM thickness and SSA grades. Finally, HRM thickness in each histological slide is theoretically assumed as representative of the HRM thickness in the entire SSA. Measuring the mean HRM thickness in each slide potentially reduced the influence of this limitation.

Our findings afford many opportunities for significant further research. Our results need to be validated in larger series. A prospective, multicenter project was recently developed and conducted at 15 European and 3 U.S. high volume centers to validate the SIB score and to assess the role and efficacy of resection technique concerning surgical, functional and oncologic outcomes after NSS. Indeed, a better understanding of which combinations of SIB scores are more prevalent among surgeons would yield potential insights into NSS care patterns. In addition, assessment of the relationship between ORS and RT, an understanding of whether tumor complexity can influence RT, and a better understanding of whether the type of RT can have an independent clinical impact on perioperative, oncologic and functional outcomes after NSS harbors potential for the improvement of kidney cancer care. To evaluate these questions high quality, prospective studies are warranted. Standardized reporting of surgical results is essential and future NSS guidelines should address this need.

\section{CONCLUSIONS}

The SIB scoring system is the first standardized reporting mechanism to communicate RT during NSS. Our study proved the applicability of the model in a real-world clinical setting and provided robust histopathological validation of its utility.

\section{REFERENCES}

1. Minervini A, Carini $M$, Uzzo RG et al: Standardized reporting of resection technique during nephron-sparing surgery: the surfaceintermediate-base margin score. Eur Urol 2014; 66: 803 .

2. Simmons MN, Hillyer SP, Lee BH et al: Functional recovery after partial nephrectomy: effects of volume loss and ischemic injury. J Urol 2012; 187: 1667.

3. Mir MC, Campbell RA, Sharma N et al: Parenchymal volume preservation and ischemia during partial nephrectomy: functional and volumetric analysis. Urology 2013; 82: 263.
4. Song C, Bang JK, Park HK et al: Factors influencing renal function reduction after partial nephrectomy. J Urol 2009; 181: 48.

5. Mukkamala A, Allam CL, Ellison JS et al: Tumor enucleation vs sharp excision in minimally invasive partial nephrectomy: technical benefit 
without impact on functional or oncologic outcomes. Urology 2014; 83: 1294.

6. Minervini A, Ficarra $V$, Rocco $F$ et al: Simple enucleation is equivalent to traditional partial nephrectomy for renal cell carcinoma: results of a nonrandomized, retrospective, comparative study. J Urol 2011; 185: 1604.

7. Mir MC, Ercole C, Takagi T et al: Decline in renal function after partial nephrectomy: etiology and prevention. J Urol 2015; 193: 1889.

8. Kutikov A, Vanarsdalen KN, Gershman B et al: Enucleation of renal cell carcinoma with ablation of the tumour base. BJU Int 2008; 102: 688 .

9. Minervini A, Campi R, Smaldone MC et al: Reply to Vincenzo Ficarra, Vito Palumbo, Afrovita Kungulli and Gianluca Giannarini's Letter to the Editor re: Andrea Minervini, Marco Carini, Robert
G. Uzzo, Riccardo Campi, Marc C. Smaldone, Alexander Kutikov. Standardized Reporting of Resection Technique During Nephron-sparing Surgery: The Surface-Intermediate-Base Margin Score. Eur Urol 2014; 66:803-5. Eur Urol 2015; 67: e48.

10. Edge SB, Byrd DR, Comptom CC et al: AJCC Staging Manual, 7th ed. Philadelphia: Springer 2009.

11. Delahunt B, Chevile JC, Martignoni $G$ et al: The International Society of Urological Pathology (ISUP) Grading System for renal cell carcinoma and other prognostic parameters. Am J Surg Pathol 2013; 37: 1490.

12. Srigley JR, Delahunt B, Eble JN et al: The International Society of Urological Pathology (ISUP)
Vancouver Classification of Renal Neoplasia. Am J Surg Pathol 2013; 37: 1469.

13. Lang TA and Altman DG: Basic statistical reporting for articles published in biomedical journals: the Statistical Analyses and Methods in the Published Literature or SAMPL guidelines. In: Science Editors' Handbook, 2nd ed. Edited by $\mathrm{P}$ Smart, $\mathrm{H}$ Maisonneuve and A Polderman. European Association of Science Editors 2013

14. Ficarra V, Palumbo V, Kungulli A et al: Re: Andrea Minervini, Marco Carini, Robert G. Uzzo, Riccardo Campi, Marc C. Smaldone, Alexander Kutikov. Standardized reporting of resection technique during nephron-sparing surgery: the surfaceintermediate-base margin score. Eur Urol 2014; 66: 803-5. Eur Urol 2015; 67: e45. 